\title{
Classification Model for Hotspot Occurrences Using Spatial Decision Tree Algorithm
}

\author{
${ }^{1,3}$ Imas Sukaesih Sitanggang, \\ ${ }^{1}$ Razali Yaakob, ${ }^{1}$ Norwati Mustapha and ${ }^{2}$ A.N. Ainuddin \\ ${ }^{1}$ Department of Computer Science, Faculty of Computer Science and Information Technology, \\ ${ }^{2}$ Institute of Tropical Forestry and Forest Products (INTROP), \\ Universiti Putra Malaysia, 43400 Serdang Selangor, Malaysia \\ ${ }^{3}$ Department of Computer Science, Faculty of Natural Science and Mathematics, \\ Bogor Agricultural University, Bogor 16680, Indonesia
}

Received 2012-04-11, Revised 2012-07-30; Accepted 2013-04-11

\begin{abstract}
Developing a predictive model for forest fires occurrence is an important activity in a fire prevention program. The model describes characteristics of areas where fires occur based on past fires data. It is essential as an early warning system for preventing forest fires, thus major damages because of fires can be avoided. This study describes the application of data mining technique namely decision tree on forest fires data. We improved the ID3 decision tree algorithm such that it can be utilized on spatial data in order to develop a classification model for hotspots occurrence. The ID3 algorithm which is originally designed for a non-spatial dataset has been improved to construct a spatial decision tree from a spatial dataset containing discrete features (points, lines and polygons). As the ID3 algorithm that uses information gain in the attribute selection, the proposed algorithm uses spatial information gain to choose the best splitting layer from a set of explanatory layers. The new formula for spatial information gain is proposed using spatial measures for point, line and polygon features. The proposed algorithm has been applied on the forest fire dataset for Rokan Hilir district in Riau Province in Indonesia. The dataset contains physical data, socio-economic, weather data as well as hotspots and non-hotspots occurrence as target objects. The result is a spatial decision tree with 276 leaves with distance from target objects to the nearest river as the first test layer and the accuracy on the training set of $87.69 \%$. Empirical result demonstrates that the proposed algorithm can be used to join two spatial objects in constructing a spatial decision tree from a spatial dataset. The algorithm results a predictive model for hotspots occurrence from the real dataset on forest fires with high accuracy on the training set.
\end{abstract}

Keywords: Spatial ID3 Algorithm, Spatial Information Gain, Forest Fires, Hotspots Occurrence

\section{INTRODUCTION}

Forest fires in various parts in Sumatera and Kalimantan, Indonesia occur every year especially in dry season. This phenomenon causes many negative effects in various aspects of life such as natural environment, economic and health. Forest fire is considered as a regional and global disaster because its effects influence not only people in local areas but also those who live in neighbouring countries such as Malaysia and Singapore. Fire prevention has an important role in minimizing the damage due to forest fires. An early warning system as one of the activities in fire prevention needs to be developed in order to assess forest fire risks.

Many studies have been conducted in developing wildfire risk models by integrating Geographical Information Systems (GISs) and remote sensing. Spatial operations in GISs have widely applied to analyze causes Universiti Putra Malaysia, 43400 Serdang Selangor, Malaysia 
of fires and their relationships as well as to produce fire risk maps. The GIS-based method of Complete Mapping Analysis (CMA) is applied in (Boonyanuphap et al., 2001) to create the wildfire risk model for the area of Sasamba in East Kalimantan in Indonesia. A model of forest fire hazard in East Kalimantan in Indonesia using the remote sensing technique integrated with the GIS has been constructed in (Darmawan et al., 2001). In other studies, GISs and remote sensing are used to analyze forest fire data to create forest fires risk models for some regions in Indonesia (Hadi, 2006; Danan, 2008). The method Complete Mapping Analysis (CMA) in (Hadi, 2006) and Multi-criteria Analysis (MCA) in (Danan, 2008) are also applied to analyse the fire risk factors and relations between the factors. A-GIS-based peat swamp forest fire hazard model that integrates Analytical Hierarchy Process (AHP) and GIS analysis was developed for the region Pekan in Pahang Malaysia (Setiawan, 2007). The model is based on five parameters: fuel type, road proximity, elevation, slope and aspect, which influence the occurrence and spread of forest fires. A GIS is utilized in fire hazard modeling and mapping of fire hazard rating in peat swamp forest of Penor/Kuantan District of Pahang in Malaysia (Sheriza, 2007). The data used in this work include fuel types, roads and canals.

Studies about forest fire risks may include huge spatial data including physical, climate and socioeconomic data. The data are stored in spatial databases in GISs. Spatial databases contain large number of spatial features and their relationships for further manipulation and analysis to help users in decision making process. Criteria evaluation and weighting methods, such as Complete Mapping Analysis (CMA) and Multi-criteria Analysis (MCA), are most applied to evaluate small problems containing few criteria. This situation has lead to the increasing in applying data mining techniques to extract interesting spatial patterns from large spatial data. Data mining tasks including association rules mining, classification and prediction, as well as cluster analysis have been successfully employed to analyse spatial data related to forest fires (Tay et al., 2003; Stojanova et al., 2007; Yu and Bian, 2007; Kalli and Ramakrishna, 2008; Hu et al., 2009).

Extracting interesting and useful patterns from spatial datasets is more difficult than those from traditional numeric and categorical data because spatial data types are complex (Shekhar et al., 2004). Furthermore, extracting patterns from spatial datasets includes spatial relationships and spatial autocorrelation (Shekhar et al., 2004). Classical data mining methods do not support locations of objects or relationships between objects that implicitly exist in a spatial dataset (Zeitouni,
2000). Therefore the methods cannot be utilized to discover knowledge from spatial datasets. Locations of objects determine relations of the objects to its neighbours. According to Koperski et al. (1998), there are three types of relation to relate an object to its neighbor, i.e., topological-relations, metric-relations and direction-relations. In order to handle spatial data and the relations to its neighbors that implicitly exist in the spatial data, new data mining methods need to be developed. One of the spatial data mining algorithms that has been introduced in many studies is spatial classification. Spatial classification methods broaden non-spatial classification methods by involving attributes of neighboring objects and their spatial relations in addition to attributes of the objects to be classified (Koperski et al., 1998; Ester et al., 1997). In a spatial classification task, we want to extract rules that split a spatial dataset consisting of classified objects into a number of classes based on non-spatial and spatial properties, as well as spatial relations of the classified objects to other objects (Koperski et al., 1998; Ester et al., 2000; Zeitouni and Chelghoum, 2001).

In this study, we extend the ID3 decision tree algorithm (Quinlan, 1986) for a non-spatial dataset such that the algorithm can be applied on a spatial dataset containing point, line and polygon features. The proposed algorithm uses information gain for spatial data, namely spatial information gain, to choose a layer as the splitting layer. Instead of using number of tuples in a partition, spatial information gain is calculated using spatial measures. Our study adopts the formula for spatial information gain as described in (Rinzivillo and Turini, 2004). The spatial measure formula is extended for the geometry type of points, lines and polygons rather than only for polygons as in (Rinzivillo and Turini, 2004). The proposed algorithm was applied to the forest fires dataset containing physical, socioeconomic and weather data as well as hotspots occurrence for Rokan Hilir district in Riau Province in Indonesia.

\section{MATERIALS AND METHODS}

\subsection{Dataset and Study Area}

The dataset for modeling hotspots occurrence contains spread and coordinates of hotspots in 2008, physical, socio-economic, as well as weather data. The study area is Rokan Hilir district in Riau Province in Indonesia (Fig. 1). Hotspots and physical data are obtained from Ministry of Environment Indonesia and National Land Agency (BPN) Riau Province respectively. 


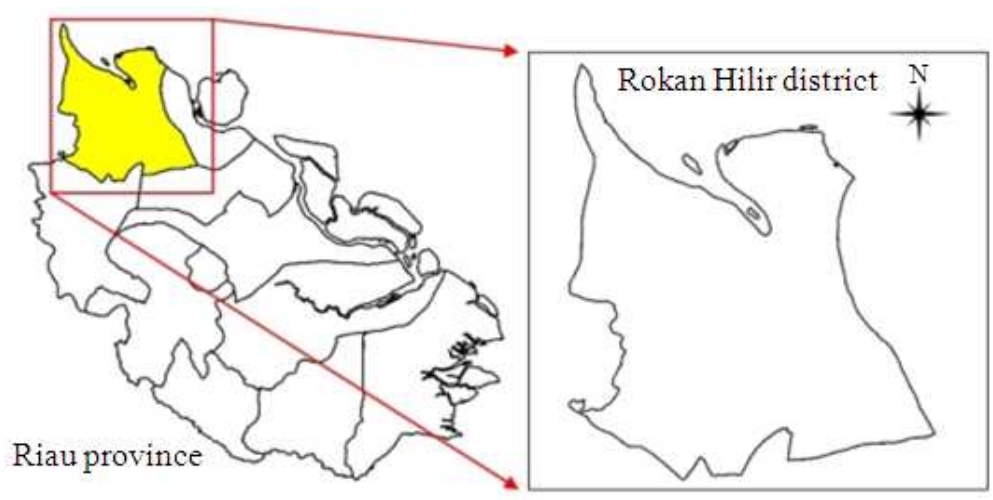

Fig. 1. Area of study

Socio-economic data are collected from StatisticsIndonesia (BPS). Weather data of 2008 including precipitation $(\mathrm{mm} /$ day), screen temperature $(\mathrm{K}), 10 \mathrm{~m}$ wind speed $(\mathrm{m} / \mathrm{s})$ and surface height $(\mathrm{m})$ are gathered from Meteorological Climatologically and Geophysical Agency (BMKG) Indonesia. There are two categories of data: spatial and non-spatial data. Non spatial data are socio-economic data for villages in Rokan Hilir which are represented in the DBF format. The data include population density and inhabitant's income source and number of schools. For mining purpose using the spatial decision tree algorithm, these non spatial data were converted to spatial data in the shp format by involving the shp files for administrative borders of villages and subdistricts in Rokan Hilir. Spatial data include physical (roads, rivers land cover and city centers) and weather data (precipitation, screen temperature, $10 \mathrm{~m}$ wind speed and surface height). We assign the spatial reference system UTM $47 \mathrm{~N}$ and datum WGS84 to all data. Preprocessing was conducted for hotspots occurrence to generate target objects, as well as for physical and socio-economic data. Data preprocessing is important to improve the quality of the data, thereby it can improve the accuracy of the resulted model as well as efficiency of data mining process. Moreover, we performed spatial interpolation using Cokriging method for weather data that are originally represented in the Net CDF format.

\subsection{Spatial Relationships}

Determining spatial relationships between two features is a major function of a Geographical Information System (GIS). Spatial relationships include topological (Egenhofer and Franzosa, 1991) such as overlap, touch and intersect as well as metric such as distance. For example, two different polygon features may be either overlap, touch, or intersect each other. Spatial relationships make spatial data mining algorithms differ from non-spatial data mining algorithms. Spatial relationships are implemented by an extension of the well-known join indices (Valduriez, 1987). The result of join index between two relations is a new relation consisting of indices pairs each referencing a tuple of each relation. The pairs of indices refer to objects that meet the join criterion. The structure Spatial Join Index (SJI) as an extension of the join indices (Valduriez, 1987 ) in the relational database framework is introduced in (Zeitouni et al., 2000). Join indices can be handled in the same way as other tables and manipulated using the standardized SQL query language (Zeitouni et al., 2000). In addition to two columns of object identifiers, a SJI has a third column that contains spatial relationship between two layers. Our study adopts the concept of SJI as in (Zeitouni et al., 2000) to store relations between two different layers in a spatial database. Instead of spatial relationship that may be either numerical or Boolean value, the quantitative values in the third column of SJI are spatial measures of features resulted from spatial relationships between two layers.

We consider an input for the algorithm a spatial dataset as a set of layers L. Each layer in L is a collection of spatial objects and has only one of the following geometric types: polygons or lines or points. Assume that each object in a layer is uniquely identified. Let $\mathrm{L}$ is a set of layers; $\mathrm{L}_{\mathrm{i}}$ and $\mathrm{L}_{\mathrm{j}}$ are two distinct layers in $\mathrm{L}$. A spatial relationship applied to $\mathrm{L}_{\mathrm{i}}$ and $\mathrm{L}_{\mathrm{j}}$ is denoted $\operatorname{SpatRel}\left(\mathrm{L}_{\mathrm{i}}, \mathrm{L}_{\mathrm{j}}\right)$ that can be topological relation or metric relation. For the case of topological relation, $\operatorname{SpatRel}\left(\mathrm{L}_{\mathrm{i}}, \mathrm{L}_{\mathrm{j}}\right)$ is a relation according to the dimension extended method proposed by (Clementini et al., 1993). Meanwhile, for the case of metric relation, $\operatorname{SpatRel}\left(\mathrm{o}_{\mathrm{i}}, \mathrm{o}_{\mathrm{j}}\right)$ is a distance relation proposed by (Ester et al., 2001), where $\mathrm{o}_{\mathrm{i}}$ is a spatial object in $\mathrm{L}_{\mathrm{i}}$ and $\mathrm{o}_{\mathrm{j}}$ is a spatial object in $\mathrm{L}_{\mathrm{j}}$.

Relations between two layers in a spatial dataset can result quantitative values such as distance 
between two points or an intersection area of two polygons. We denote these values as spatial measures as in (Rinzivillo and Franco, 2004) which is used in calculating spatial information gain in the proposed algorithm. For the case of topological relation, the spatial measure of a feature is defined as follows. Let $\mathrm{L}_{\mathrm{i}}$ and $\mathrm{L}_{\mathrm{j}}$ in a set of layers $L, L_{i} \neq L_{j}$, for each feature $r_{i}$ in $R=$ $\operatorname{SpatRel}\left(\mathrm{L}_{\mathrm{i}}, \mathrm{L}_{\mathrm{j}}\right)$, a spatial measure of $\mathrm{r}_{\mathrm{i}}$ denoted by SpatMes $\left(r_{i}\right)$ is defined as:

- Area of $\mathrm{r}_{\mathrm{i}}$, if $<\mathrm{L}_{\mathrm{i}}$ in $\mathrm{L}_{\mathrm{j}}>$ or $<\mathrm{L}_{\mathrm{i}}$ overlap $\mathrm{L}_{\mathrm{j}}>$ hold for all features in $\mathrm{L}_{\mathrm{i}}$ and $\mathrm{L}_{\mathrm{j}}$ represented in polygons

- Count of $r_{i}$, if $<\mathrm{L}_{\mathrm{i}}$ in $\mathrm{L}_{\mathrm{j}}>$ holds for all features in $\mathrm{L}_{\mathrm{i}}$ represented in points and all features in $L_{j}$ represented in polygons

For the case of metric relation, we define a distance function from $\mathrm{p}$ to $\mathrm{q}$ as $\operatorname{dist}(\mathrm{p}, \mathrm{q})$, distance from a point (or line) $\mathrm{p}$ in $\mathrm{L}_{\mathrm{i}}$ to a point (or line) $\mathrm{q}$ in $\mathrm{L}_{\mathrm{j}}$.

Spatial measure of $\mathrm{R}$ is denoted by $\operatorname{SpatMer}(\mathrm{R})$ and defined as Equation 1:

$$
\begin{aligned}
& \operatorname{SpatMes}(R)=f\left(\operatorname{SpatMes}\left(r_{1}\right)\right. \\
& \left.\operatorname{SpatMes}\left(r_{2}\right), \ldots, \operatorname{SpatMes}\left(r_{n}\right)\right)
\end{aligned}
$$

for $r_{i}$ in $R, i=1,2, \ldots, n$ and $n$ number of features in $R$. $f$ is an aggregate function that may be either sum, min, max or average.

A spatial relationship applied to $\mathrm{L}_{\mathrm{i}}$ and $\mathrm{L}_{\mathrm{j}}$ in $\mathrm{L}$ results a new layer R. We define a Spatial Join Relation (SJR) for all features $p$ in $L_{i}$ and $q$ in $L_{j}$ as follows (Equation 2):

$$
\operatorname{SJR}=\{(\mathrm{p}, \operatorname{SpatMes}(\mathrm{r}), \mathrm{q} \mid \mathrm{r} \text { is a }
$$

feature in $\mathrm{R}$ associated to $\mathrm{pand} q\}$

\subsection{An Extended ID3 Algorithm for Spatial Data}

A spatial dataset is composed of a set of layers in which all features in a layer have the same geometry type. This study considers only discrete features including points, lines and polygons. There are two groups of layers: explanatory layers and one target layer (or reference layer) where spatial relationships are applied to construct a set of tuples. The target layer has some attributes including a target attribute that store target classes. Each explanatory layer has several attributes. One of the attributes is a predictive attribute that classify tuples in the dataset to target classes. In this study the target attribute and predictive attributes are categorical. Features (polygons, lines or points) in the target layer are related to features in explanatory layers to create a set of tuples in which each value in a tuple corresponds to value of these layers. Two distinct layers are associated to produce a new layer using a spatial relationship. A relation between two layers produces a spatial measure (Equation 1) for the new layer. Spatial measures are used in the formula for spatial information gain.

Building a spatial decision tree follows the basic learning process in the algorithm ID3 (Quinlan, 1986). The ID3 calculates information gain to define the best splitting layer for the dataset. In the spatial decision tree algorithm, we define spatial information gain to select an explanatory layer $\mathrm{L}$ that gives best splitting the spatial dataset according to values of predictive attribute in the layer L. For this purpose, as in (Rinzivillo and Turini, 2004), we apply the spatial measure (Equation 1) to the formula.

Let a dataset $D$ be a training set of class-labelled tuples. In the non-spatial decision tree algorithm, we calculate probability that an arbitrary tuple in $\mathrm{D}$ belong to class $C_{i}$ and it is estimated by $\left|C_{i, D}\right| /|D|$ where $|D|$ is number of tuples in $\mathrm{D}$ and $\left|\mathrm{C}_{\mathrm{i}, \mathrm{D}}\right|$ is number of tuples of class $C_{i}$ in D (Han and Kamber, 2001). In this study, a dataset contains some layers including a target layer which stores class labels. All objects in the target layer are represented in points. Number of tuples in the dataset is the same as number of objects in the target layer because each tuple is created by relating features in the target layer to features in explanatory layers. One feature in the target layer associates with exactly one tuple in the dataset. For simplicity we use number of objects in the target layer instead of using number of tuples in the spatial dataset in the formula of spatial entropy (Equation 3). Furthermore, in a non-spatial dataset, target classes are discrete-valued and unordered (categorical) and explanatory attributes are categorical or numerical. In spatial dataset, features in layers are represented in a particular geometric type (polygons, lines or points) that have quantitative measurements such as area and distance. Therefore we calculate spatial measures of layers (Equation 1) to replace number of tuples in a non-spatial dataset.

\subsection{Entropy}

Let a target attribute $\mathrm{C}$ in a target layer $\mathrm{S}$ has 1 distinct classes (i.e., $\mathrm{c}_{1}, \mathrm{c}_{2}, \ldots, \mathrm{c}_{1}$ ), entropy for $\mathrm{S}$ represents the expected information needed to determine the class of tuples in the dataset and defined as Equation 3:

$\mathrm{H}(\mathrm{S})=-\sum_{\mathrm{i}=1}^{1} \frac{\operatorname{SpatMes}\left(\mathrm{S}_{\mathrm{c}_{\mathrm{i}}}\right)}{\operatorname{SpatMes}(\mathrm{S})} \log _{2} \frac{\operatorname{SpatMes}\left(\mathrm{S}_{\mathrm{c}_{\mathrm{i}}}\right)}{\operatorname{SpatMes}(\mathrm{S})}$

SpatMes(S) represents the spatial measure of layer $\mathrm{S}$ as defined in (Equation 1). 


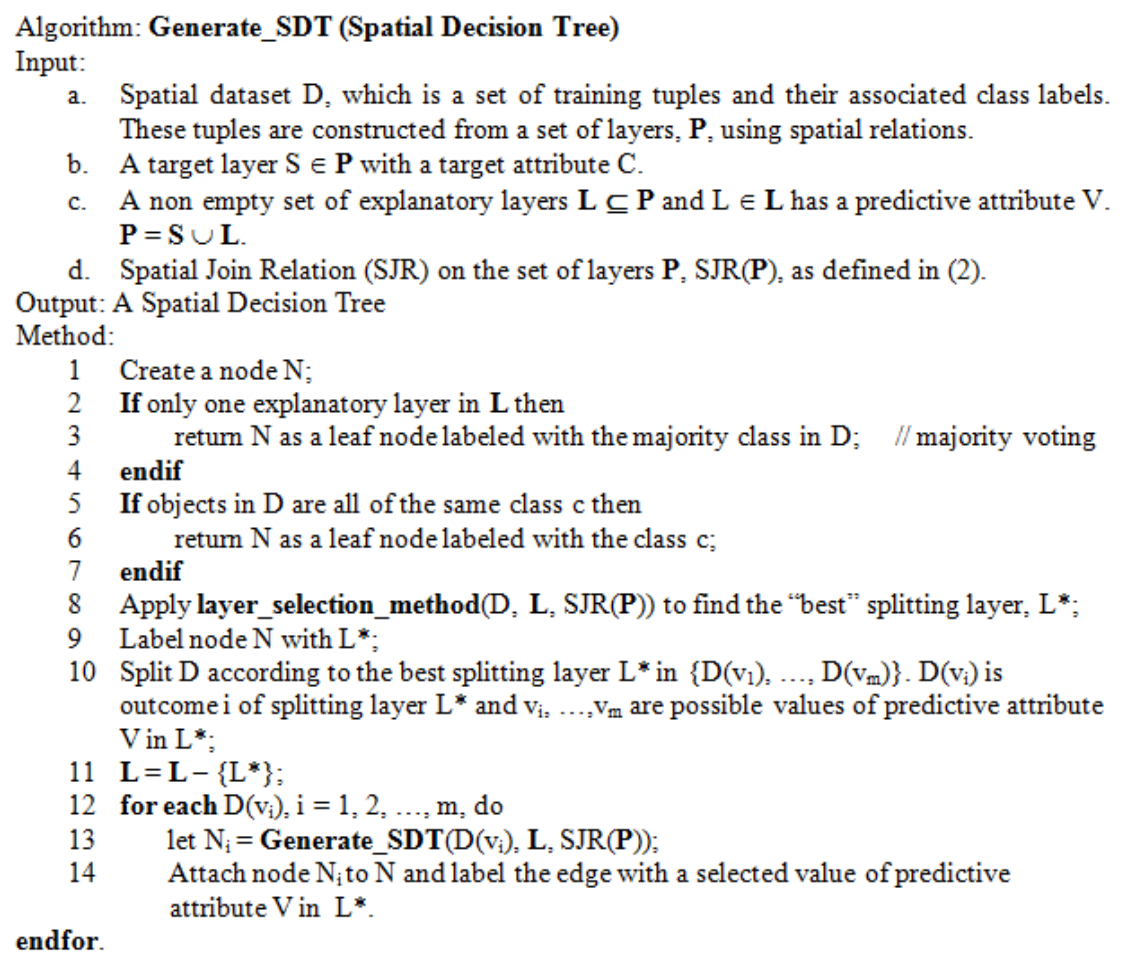

Fig. 2. Extended ID3 decision tree algorithm (Sitanggang et al., 2011)

Let an explanatory attribute $\mathrm{V}$ in an explanatory (non-target) layer $\mathrm{L}$ has $\mathrm{q}$ distinct values (i.e., $\mathrm{v}_{1}, \mathrm{v}_{2}, \ldots$, $\mathrm{v}_{\mathrm{q}}$ ). We partition the objects in target layer $\mathrm{S}$ according to the layer $\mathrm{L}$ then we have a set of layers $\mathrm{L}\left(\mathrm{v}_{\mathrm{i}}, \mathrm{S}\right)$ for each possible value $\mathrm{v}_{\mathrm{i}}$ in $\mathrm{L}$. In our study, we assume that the layer L covers all areas in the layer $\mathrm{S}$. The expected entropy value for splitting is given by Equation 4:

$$
\mathrm{H}(\mathrm{S} \mid \mathrm{L})=\sum_{\mathrm{j}=1}^{\mathrm{q}} \frac{\operatorname{SpatMes}\left(\mathrm{L}\left(\mathrm{v}_{\mathrm{j}}, \mathrm{S}\right)\right)}{\operatorname{SpatMes}(\mathrm{S})} \mathrm{H}\left(\mathrm{L}\left(\mathrm{v}_{\mathrm{j}}, \mathrm{S}\right)\right)
$$

$\mathrm{H}(\mathrm{S} \mid \mathrm{L})$ represents the amount of information needed (after the partitioning) in order to arrive at an exact classification.

\subsection{Spatial Information Gain}

The spatial information gain for the layer $\mathrm{L}$ is given by Equation 5:

$$
\operatorname{Gain}(\mathrm{L})=\mathrm{H}(\mathrm{S})-\mathrm{H}(\mathrm{S} \mid \mathrm{L})
$$

Gain(L) denotes how much information that would be gained by branching on the layer $\mathrm{L}$. The layer $\mathrm{L}$ with the highest information gain, $(\operatorname{Gain}(\mathrm{L}))$, is chosen as the splitting layer at a node N. Objects in the dataset are partitioned according to the layer $\mathrm{L}$ to result the "best classification" such that $\mathrm{H}(\mathrm{S} \mid \mathrm{L})$ minimum.

\subsection{Spatial Decision tree Algorithm}

Figure 2 shows our proposed algorithm to generate a Spatial Decision Tree (SDT) as discussed in our previous work (Sitanggang et al., 2011). Inputs of the algorithm are divided into two groups: (1) a set of layers containing some explanatory layers and one target layer that hold class labels for tuples in the dataset and (2) Spatial Join Relations (SJRs) storing spatial measures for features resulted from spatial relations between two layers. The algorithm generates a tree by selecting the best layer to separate the dataset into smaller partitions as pure as possible meaning that all tuples in partitions belong to the same class.

\section{RESULTS}

The proposed algorithm has been applied to the real active fires dataset for the Rokan Hilir District in Riau Province Indonesia with the total area of $8,881.59 \mathrm{~km}^{2}$. The dataset contains ten explanatory layers and one target layer. The target layer consists of active fires (hotspots) as true alarm data and non-hotspots as false alarm data. 
Table 1. Number of features and distinct values in the dataset

\begin{tabular}{lll}
\hline Layer & Number of features & Number of distinct values \\
\hline Physical & & \\
Distance to nearest river (dist_river) & 744 points & 3 (low, medium, high) \\
$\begin{array}{l}\text { Distance to nearest road (dist_road) } \\
\text { Distance to nearest city center(dist_city) }\end{array}$ & 744 points & 3 (low, medium, high) \\
Land cover (land_cover) & 744 points & 3 (low, medium, high) \\
Social-economy & 3107 polygons & 12 (Dryland_forest, plantation, Water_body and so on) \\
Income source (income_source) & 117 polygons & 7 (Forestry, Agriculture, Trading_restaurant and so on) \\
Population density (population) & 117 polygons & 3 (low, medium, high) \\
Number of school (school) & 117 polygons & 3 (low, medium, high) \\
Weather & & \\
Precipitation in mm/day (precipitation) & 7 polygons & 2,3 \\
Screen temperature in K (screen_temp) & 7 polygons & $297,298,299$ \\
$10 \mathrm{~m}$ wind speed in m/s (wind_speed) & 7 polygons & $0,1,2$ \\
Target & & \\
Target & 744 points & \\
\hline
\end{tabular}

False alarm data were randomly generated and they are located within an area at least $1 \mathrm{~km}$ away from any true alarm data. Explanatory layers are grouped into three groups i.e., physical, socio-economy and weather.

Table 1 summaries the number of features in the dataset for each layer. Spatial relationship $I n$ is applied to the target layer and explanatory layers represented in polygon (land_cover, income_source, population, school, precipitation, screen_temp and wind_speed) to result spatial measure $\bar{C}$ ount. Additionally, the spatial relationship Distance is calculated to relate the target layer to the river, road and city centre layer. We use the aggregate function Min to determine distance from target objects to nearest river, road and city centre. The aggregate function Sum is applied to extract all objects in the target layer which are located inside polygon features.

The decision tree generated from the proposed spatial decision tree algorithm contains 276 leaves with the accuracy of $87.69 \%$. The first test attribute of the tree is distance from target objects to nearest river. Below are some rules generated from the tree:

- IF dist_river $(\mathrm{km})>3$ AND $0 \leq$ wind_speed $(\mathrm{m} / \mathrm{s})<$ 1 AND income_source $=$ Forestry AND dist_road $(\mathrm{km}) \leq 2.5$ AND dist_city $(\mathrm{km})>14$ THEN hotspot occurrence $=\mathrm{T}$

- IF dist_river $(\mathrm{km})>3$ AND $0 \leq$ wind_speed $(\mathrm{m} / \mathrm{s})<$ 1 AND income_source $=$ Plantation AND land_cover $=$ Shrubs $\bar{s}$ AND dist_city $(\mathrm{km}) \leq 7$ THEN hotspot occurrence $=\mathrm{F}$

- IF dist_river $(\mathrm{km})>3$ AND $1 \leq$ wind_speed $(\mathrm{m} / \mathrm{s})<$ 2 AND $50<$ population_density $\leq 100$ AND land_cover $=$ Shrubs AND income_source = Other_agriculture THEN hotspot occurrence $=\mathrm{F}$
- $\quad$ IF $1.5<$ dist_river $(\mathrm{km}) \leq 3$ AND income_source $=$ Forestry AND dist_city $(\mathrm{km})>14$ THEN hotspot occurrence $=\mathrm{T}$

- IF dist_river $(\mathrm{km}) \leq 1.5$ AND land_cover = Bare_land AND dist_city $(\mathrm{km})>14$ AND dist_road $(\mathrm{km}) \leq 2.5$ THEN hotspot occurrence $=\mathrm{T}$

\section{DISCUSSION}

The proposed algorithm performs well on the spatial dataset containing polygon, line and point features to classify 744 target objects (hotspots and non-hotspots) with the accuracy of $87.69 \%$. Based on the experiment on the real forest fires data, the proposed algorithm can be used to join two spatial objects in constructing a spatial decision tree from a spatial dataset. From the tree we can generate 276 rules in which the first test condition for each rule is distance from a target object to nearest river.

The spatial dataset may contain noises and outliers that may result the problem of over fitting. We will implement the tree pruning method for the resulted tree to overcome the problem such that the tree becomes simpler and has higher accuracy. Due to non-availability a real testing dataset, the accuracy was calculated based on the training dataset. Therefore, in the future we will apply the tree to another area as a testing spatial dataset to study the performance of the proposed algorithm on a new area.

\section{CONCLUSION}

This study presents an extended ID3 algorithm to create a classifier namely spatial decision tree from spatial data. For mining purpose, a spatial dataset is 
organized in a set of layers in which the layers are grouped into two categories i.e., explanatory layers and a target layer. All layers are represented in discrete features (polygons, lines and points). The algorithm calculates spatial information gain as the extension of information gain in the non-spatial ID3 algorithm. Spatial measure resulted from the spatial relationships that may be either topological or metric (distance) is used in the formula of spatial information gain instead of number of tuples in the non-spatial information gain. The algorithm selects an explanatory layer which has the highest spatial information gain as the best splitting layer. This layer separates the dataset into smaller partitions as pure as possible such that all tuples in partitions belong to the same class. The extended ID3 algorithm has been applied on the real spatial dataset on forest fires consisting of ten explanatory layers and a target layer. Empirical result shows that the algorithm can be used to join two spatial objects in constructing spatial decision trees. The result is a spatial decision tree consisting of the root (distance from target objects to the nearest river) and 276 leaves. The accuracy of the tree on the training set is $87.69 \%$.

\section{ACKNOWLEDGMENT}

The researchers would like to thank Indonesia Directorate General of Higher Education (IDGHE), Ministry of National Education, Indonesia for supporting PhD Scholarship (Contract No. 1724.2/D4.4/2008) and Southeast Asian Regional Center for Graduate Study and Research in Agriculture (SEARCA) for partially supporting the research.

\section{REFERENCES}

Boonyanuphap, J., F.G. Suratmo, I.N.S. Jaya and F. Amhir, 2001. GIS-based method in developing wildfire risk model (case study in Sasamba, East Kalimantan, Indonesia). J. Manajemen Hutan Tropika, 7: 33-45.

Clementini, E., P.D. Felice and P.V. Oosterom, 1993. A small set of formal topological relationships suitable for end-user interaction. Proceedings of the Third International Symposium on Advances in Spatial Databases, (ASD' 93), Springer-Verlag London, UK., pp: 277-295.

Danan, P.H., 2008. A RS/GIS-Based Multi-Criteria Approaches to Assess Forest Fire Hazard in Indonesia (Case Study: West Kutai District, East Kalimantan Province). MSc Thesis, Bogor Agricultural University.
Darmawan, M., M. Aniya and S. Tsuyuki, 2001. Forest fire hazard model using remote sensing and geographic information systems: Toward understanding of land and forest degradation in lowland areas of East Kalimantan, Indonesia. Proceedings of the 22nd Asian Conference on Remote Sensing, Nov. 5-9, Singapore.

Egenhofer, M.J. and R.D. Franzosa, 1991. Point-set topological spatial relations. Int. J. Geographical Inform. Syst., 5: 161-174. DOI: 10.1080/02693799108927841

Ester, M., A. Frommelt, H.P. Kriegel and J. Sander, 2000. Spatial data mining: Database primitives, algorithms and efficient DBMS support. Data Min. Knowl. Dis., 4: 193-216. DOI: 10.1023/A:1009843930701

Ester, M., H. P. Kriegel and J. Sander, 2001. Algorithms and Applications for Spatial Data Mining. In: Geographic Data Mining and Knowledge Discovery, Miller H.J. and J. Han (Eds.), Taylor and Francis, London, ISBN-10: 0415233690, pp: 1-32.

Ester, M., H.P. Kriegel and J. Sander, 1997. Spatial data mining: A database approach. Proceedings of the 5th International Symposium on Large Spatial Databases, Jul. 15-18, Springer Berlin Heidelberg, pp: 47-66. DOI: 10.1007/3-540-63238-7_24

Hadi, M., 2006. Pemodelan spasial kerawanan kebakaran di lahan gambut: Studi kasus kabupaten ben gkalis, provinsi riau. MSc Thesis, Bogor Agricultural University.

Han, J. and M. Kamber, 2001. Data Mining: Concepts and Techniques. 7th Edn., Morgan Kaufmann, San Francisco, ISBN: ISBN-10: 1558604898, pp: 550.

Hu, L., G. Zhou and Y. Qiu, 2009. Application of Apriori algorithm to the data mining of the wildfire. Proceedings of the 6th International Conference on Fuzzy Systems and Knowledge Discovery, Aug. 1416, IEEE Xplore Press, Tianjin, pp: 426-429. DOI: 10.1109/FSKD.2009.228

Kalli, S.N.P. and S. Ramakrishna, 2008. An autonomous forest fire detection system based on spatial data mining and fuzzy logic. Int. J. Comput. Sci. Net. Sec., 8: 49-55.

Koperski, K., J. Han and N. Stefanovic, 1998. An efficient two-step method for classification of spatial data. Proceedings of International Symposium on Spatial Data Handling, (SDH' 98).

Quinlan, J.R., 1986. Induction of decision trees. Mach. Learn., 1: 81-106. DOI: 10.1007/BF00116251 
Rinzivillo, S. and F. Turini, 2004. Classification in geographical information systems. Proceedings of the 8th European Conference on Principles and Practice of Knowledge Discovery in Databases, (KDD, 04), Springer-Verlag New York, Inc., New York, USA., pp: 374-385.

Setiawan, I., 2007. Development of a GIS based peat swamp forest fire hazard model. Ph.D Thesis, Universiti Putra Malaysia.

Shekhar, S., P. Zhang and Y. Huang, 2004. Trends in spatial data mining. Science, 40: 363-363. DOI: 10.1007/0-387-25465-X_39

Sheriza, M.R., 2007. Forest fire hazard rating assessment in peat swamp forest using integrated remote sensing and geographical information system. MSc Thesis, Universiti Putra Malaysia.

Sitanggang, I.S., R. Yaakob, N. Mustapha and A.A.B. Nuruddin, 2011. An extended ID3 decision tree algorithm for spatial data. Proceedings of the IEEE International Conference on Spatial Data Mining and Geographical Knowledge Services, Jun. 29-Jul. 1, IEEE Press, pp: 48-53.

Stojanova, D., P. Panov, A. Kobler, S. Dzeroski and K. Taskova, 2007. Learning to predict forest fires with different data mining techniques. Pennsylvania State University.

Tay, S.C., W. Hsu, K.H. Lim and L.C. Yap, 2003. Spatial data mining: Clustering of hot spots and pattern recognition. Proceedings of the IEEE International Geoscience and Remote Sensing Symposium, Jul. 21-25, IEEE Xplore Press, pp: 3685-3687. DOI: 10.1109/IGARSS.2003.1295237
Valduriez, P., 1987. Join indices. ACM Trans. Database Syst., 12: 218-246. DOI: 10.1145/22952.22955

$\mathrm{Yu}, \mathrm{L}$. and F. Bian, 2007. An incremental data mining method for spatial association rule in GIS based fireproof system. Proceedings of the International Conference on Wireless Communications, Networking and Mobile Computing, Sept. 21-25, IEEE Xplore Press, Shanghai, pp: 5983-5986. DOI: 10.1109/WICOM.2007.1467

Zeitouni, K. and N. Chelghoum, 2001. Spatial decision tree-application to traffic risk analysis. Proceedings of the ACS/IEEE International Conference on Computer Systems and Applications, Jun. 25-29, IEEE Xplore Press, Beirut, pp: 203-207. DOI: 10.1109/AICCSA.2001.933978

Zeitouni, K., 2000. A survey of spatial data mining methods databases and statistics point of views. Proceedings of the Information Resources Management Association International Conference on Challenges of Information Technology Management in the 21st Century, (ITMC, 00), IGI Publishing Hershey, PA, USA., pp: 487-491.

Zeitouni, K., L. Yeh and M.A. Aufaure, 2000. Join indices as a tool for spatial data mining. Proceedings of the 1st International Workshop on Temporal, Spatial and Spatio-Temporal Data Mining-Revised Papers, (MRP' 00), Springer-Verlag London, UK., pp: 105-116. 